\title{
Intravitreal Devices for the Treatment of Vitreous Inflammation
}

\author{
John B. Christoforidis, ${ }^{1}$ Susie Chang, ${ }^{2}$ Angela Jiang, ${ }^{2}$ Jillian Wang, ${ }^{2}$ and Colleen M. Cebulla ${ }^{2}$ \\ ${ }^{1}$ Retina Division, Department of Ophthalmology, The Ohio State University Wexner Medical Center, Columbus, OH 43212, USA \\ ${ }^{2}$ Department of Ophthalmology, The Ohio State University Wexner Medical Center, Columbus, OH 43212, USA
}

Correspondence should be addressed to John B. Christoforidis, jbchristo@hotmail.com

Received 14 June 2012; Accepted 31 July 2012

Academic Editor: Mario R. Romano

Copyright ( $) 2012$ John B. Christoforidis et al. This is an open access article distributed under the Creative Commons Attribution License, which permits unrestricted use, distribution, and reproduction in any medium, provided the original work is properly cited.

The eye is a well-suited organ for local delivery of therapeutics to treat vitreous inflammation as well as other pathologic conditions that induce visual loss. Several conditions are particularly challenging to treat and often require chronic courses of therapy. The use of implantable intravitreal devices for drug delivery is an emerging field in the treatment of vitreous inflammation as well as other ophthalmologic diseases. There are unique challenges in the design of these devices which include implants, polymers, and micro- and nanoparticles. This paper reviews current and investigational drug delivery systems for treating vitreous inflammation as well as other pathologic conditions that induce visual loss. The use of nonbiodegradable devices such as polyvinyl alcohol-ethylene vinyl acetate polymers and polysulfone capillary fibers, and biodegradable devices such as polylactic acid, polyglycolic acid, and polylactic-co-glycolic acid, polycaprolactones, and polyanhydrides are reviewed. Clinically used implantable devices for therapeutic agents including ganciclovir, fluocinolone acetonide, triamcinolone acetonide, and dexamethasone are described. Finally, recently developed investigational particulate drug delivery systems in the form of liposomes, microspheres, and nanoparticles are examined.

\section{Introduction}

The eye is a model organ for the implantation of devices that provide long-lasting infusion of a therapeutic agent. It is easily accessible for implantation of such a device and success of therapy is measurable objectively by direct visualization of the intraocular structures and by patient responsiveness. The treatment of posterior uveitis and vitreous inflammation usually involves a chronic course of therapy often over a period of years. Topical agents require frequent administration which is often impractical for patients. Sub-Tenon's and intravitreal steroid injections also can require frequent retreatment to adequately control disease. Treatment with powerful systemic corticosteroid and immunomodulatory agents most often have poor vitreous penetration and can be associated with severe systemic side effects [1]. Implantable devices offer an alternative therapeutic approach that can circumvent many challenges of these other modes of therapy.

The first implantable device for clinical use was developed in 1992 [2]. Vitrasert, a nonbiodegradable implant, delivers ganciclovir into the eye for the treatment of acquired-immunodeficiency-syndrome (AIDS)-related cytomegalovirus (CMV). Newer biodegradable implantable devices can provide sustained release of pharmacologics. More recently, there are serious investigations of biodegradable polymers that encapsulate particulate systems for longlasting delivery of therapeutic nanoparticles which can be injected intravitreally.

In this review, current and investigational drug delivery systems for treating vitreous inflammation are described. These are summarized in Tables 1 and 2.

\section{Drug Delivery Implant Polymers}

2.1. Nonbiodegradable Devices. Nonbiodegradable devices require surgical implantation and contain a drug reservoir within a permeable polymer membrane. Although useful in some clinical settings, nonbiodegradable implants are not without limitations. Due to their large size a relatively large incision is required for implantation. Furthermore, these devices typically require removal and reimplantation of a second device once the drug has been completely released. 
TABLE 1: Drug delivery implant polymers.

\begin{tabular}{|c|c|c|}
\hline Material & Properties & Clinical application \\
\hline \multicolumn{3}{|c|}{ Nonbiodegradable devices } \\
\hline Ethylene vinyl acetate & Nonpermeable, hydrophobic & $\begin{array}{l}\text { Vitrasert implant, intravitreal dexamethasone, and } \\
\text { cyclosporine }\end{array}$ \\
\hline Polysulfone capillary fiber & $\begin{array}{l}\text { Water impermeable; increases surface area for drug } \\
\text { release }\end{array}$ & $\begin{array}{l}\text { Used experimentally for carboxyfluorescein dye release } \\
\text { and daunomycin in rabbit eyes }\end{array}$ \\
\hline Polyvinyl alcohol & Permeable & $\begin{array}{l}\text { Vitrasert implant, intravitreal dexamethasone, and } \\
\text { cyclosporine }\end{array}$ \\
\hline \multicolumn{3}{|c|}{ Biodegradable devices } \\
\hline Polyanhydrides & $\begin{array}{l}\text { Degrade by surface erosion into biocompatible } \\
\text { monomers }\end{array}$ & 5-fluorouracil, taxol, and etoposide \\
\hline Polycaprolactone & Semicrystalline, hydrophobic & $\begin{array}{l}\text { 5-fluorouracil, dexamethasone, and triamcinolone } \\
\text { implants }\end{array}$ \\
\hline Polyglycolic acid & Semicrystalline; synthesized using toxic solvents & \\
\hline Polylactic acid & $\begin{array}{l}\text { Hydrophobic; degrades more slowly than } \\
\text { polyglycolic acid }\end{array}$ & \\
\hline Polylactic-co-glycolic acid & $\begin{array}{l}\text { Copolymer (adjustable ratio) of polyglycolic and } \\
\text { polylactic acid }\end{array}$ & Dexamethasone (Ozurdex), indomethacin \\
\hline
\end{tabular}

TABLE 2: Characteristics of intravitreal devices.

\begin{tabular}{|c|c|c|c|c|}
\hline Device & Materials & Active agent & $\begin{array}{l}\text { Duration of } \\
\text { drug release }\end{array}$ & Diseases \\
\hline \multicolumn{5}{|c|}{ Nonbiodegradable devices } \\
\hline I-vation & $\begin{array}{l}\text { Drug-polymer-coated } \\
\text { nonferrous alloy helix (polybutyl } \\
\text { methacrylate/polyvinyl alcohol; } \\
\text { bravo drug delivery polymer } \\
\text { matrix) }\end{array}$ & $\begin{array}{l}\text { Triamcinolone acetonide } \\
(1-3 \mu \mathrm{g} / \text { day })\end{array}$ & 2 years & $\begin{array}{l}\text { Investigational: diabetic macular } \\
\text { edema phase } 2 \mathrm{~b} \text { trial suspended in } \\
2008\end{array}$ \\
\hline Illuvien/medidur & $\begin{array}{l}\text { Polyvinyl alcohol (with silicone } \\
\text { bioadhesive in low-dose version) }\end{array}$ & $\begin{array}{l}\text { Fluocinolone acetonide } \\
(0.59 \mathrm{mg} ; 0.2-0.5 \mu \mathrm{g} / \mathrm{day})\end{array}$ & $\begin{array}{l}18-30 \\
\text { months }\end{array}$ & $\begin{array}{l}\text { Investigational: diabetic macular } \\
\text { edema (phase } 3 \text { ) }\end{array}$ \\
\hline Retisert & Silicone/polyvinyl alcohol & $\begin{array}{l}\text { Fluocinolone acetonide } \\
(0.59 \mathrm{mg})\end{array}$ & Up to 3 years & $\begin{array}{l}\text { FDA approved for the treatment of } \\
\text { uveitis. Investigational: diabetic } \\
\text { macular edema, retinal vein } \\
\text { occlusion }\end{array}$ \\
\hline Vitrasert & EVA/polyvinyl alcohol & Ganciclovir (4.5 mg) & 5 to 8 months & Implantable reservoir system \\
\hline \multicolumn{5}{|c|}{ Biodegradable devices } \\
\hline Ozurdex & Polylactic-co-glycolic acid & Dexamethasone (0.7 mg) & 6 months & $\begin{array}{l}\text { DA approved for the treatment of } \\
\text { macular edema following branch or } \\
\text { central retinal vein occlusion. } \\
\text { Investigational: diabetic macular } \\
\text { edema, uveitis }\end{array}$ \\
\hline Surodex & $\begin{array}{l}\text { Polylactic-co-glycolic acid, } \\
\text { hydroxypropyl methylcellulose }\end{array}$ & Dexamethasone $(60 \mu \mathrm{g})$ & $7-10$ days & $\begin{array}{l}\text { Investigational in the USA: } \\
\text { postoperative inflammation } \\
\text { following cataract surgery (phase } \\
\text { 3). Regulatory approvals in } \\
\text { Singapore, China, Mexico }\end{array}$ \\
\hline
\end{tabular}

The rate of drug release can be slowed by decreasing the surface area or increasing the thickness of the permeable membrane [3]. Complications associated with these devices include retinal detachment, vitreous hemorrhage, epiretinal membrane formation, and dissolution of the implant $[4]$.
2.1.1. Polyvinyl Alcohol-Ethylene Vinyl Acetate Polymers. These devices are made of polyvinyl alcohol (PVA), a permeable polymer functioning as a structural component, and ethylene vinyl acetate (EVA), a nonpermeable hydrophobic polymer used to restrict drug release. These devices are essentially inert, almost devoid of intraocular inflammatory 
response when implanted, but must be removed to prevent fibrous encapsulation after drug delivery is complete. The initial device of this type was originally formulated to contain 5-fluorouracil and placed subconjuctivally to prevent scarring following glaucoma filtration [5]. Subsequent devices have been used for intravitreal dexamethasone and cyclosporine implantation [6, 7]. The major clinical application of this device type was the ganciclovir (Vitrasert, Bausch and Lomb) implant, which was used frequently prior to the development of highly active antiretroviral therapy for HIV.

2.1.2. Polysulfone Capillary Fiber. These devices are water impermeable and contain deep macrovoids which increase the surface area for drug release. It is permeable to both lipophilic and hydrophilic compounds and is well tolerated [7]. Polysulfone capillary fiber implants have only been used experimentally for carboxyfluorescein dye release and daunomycin in rabbit eyes $[8,9]$.

2.2. Biodegradable Devices. Biodegradable devices are particularly useful as intraocular implants since they do not need to be removed and have increased flexibility in their shape. They can be formulated as rods, discs, and microparticles [3].

\subsubsection{Polylactic Acid, Polyglycolic Acid, and Polylactic-Co-} Glycolic Acid. Polylactic acid (PLA), polyglycolic acid (PGA), and polylactic-co-glycolic acid (PLGA) are the most studied synthetic biodegradable polymers. They are biocompatible, biodegradable and are FDA approved for drug delivery [10]. These polymers are widely used as suture materials, bone screws and pins, vascular grafts and stents, and surgical scaffolds for tissue regeneration.

PGA is a semicrystalline polymer that is synthesized using toxic solvents limiting its potential for clinical use since any residual solvent may react with the drug or tissue [11]. PLA is a hydrophobic polymer that degrades more slowly than PGA. PLGA is a copolymer of PLA and PGA and is the most widely used biodegradable polymer for drug delivery. The ratio of PLA to PGA can be adjusted to modulate the rate of polymer degradation. The rate of drug release depends on the total surface area of the device, the percentage of loaded drug, the water solubility of the drug, and the speed of polymer degradation for human immunodeficiency virus [12].

There are three phases of drug release in these types of polymers.

(1) Initial burst from the surface of the implant.

(2) Diffusion phase during biodegradation of the polymer.

(3) Final burst from the disintegration of the implant.

The initial burst is followed by a longer steady drug release and is well suited for diseases that require an initial loading dose followed by tapering. However, the first and last phases release higher drug concentrations and potential toxic effects at these levels need to be considered. Blending polymers with different molecular weights can reduce the final drug burst and result in a more stable agent release
$[13,14]$. Examples of PLGA devices include dexamethasone (Ozurdex) and indomethacin.

2.2.2. Polycaprolactones. Polycaprolactone (PCL) is a polymer of $\mathcal{E}$-caprolactone, a semi-crystalline and hydrophobic compound that is biodegradable and biocompatible. It is widely used in the biomedical industry (e.g., Monocryl suture, artificial skin, and osteosynthetic material). It is very slowly degraded in the human body by hydrolysis of its ester linkages and its fragments are phagocytized [15]. When the implants are immersed in water, there is dissolution leaving pores in the PCL, allowing for a longterm, well-controlled steady release rate over a period of greater than one year $[16,17]$. Intravitreal PCL implants with 5-fluorouracil has been investigated for the prevention of proliferative vitreoretinopathy [18]. Intravitreally-placed PCL devices containing dexamethasone delivers the drug in a controlled and prolonged manner for at least 55 weeks. At 55 weeks, $79 \%$ of drug was still present in the implant. It was found to be very well tolerated in rabbit eyes with no sign of anterior or posterior segment inflammation [19]. PCL devices containing triamcinolone acetate have also been implanted in the subretinal space of rabbit eyes and was found to be well- tolerated by retinal tissue, releasing the drug for at least 4 weeks without an inflammatory response [20]. PCL can also be mixed with other polymers, usually more hydrophilic than PCL, to form copolymers which degrade at faster rates. These have been used experimentally for drug delivery of cyclosporine and tacrolimus [21, 22].

2.2.3. Polyanhydrides. Polyanhydrides are a class of biodegradable polymers that degrade by surface erosion into biocompatible monomers that are then metabolized and removed from the body [23]. Surface erosion provides a more controlled drug release compared to drugs that are released by bulk erosion, making them useful as drug delivery devices. There are several classes of polyanhydrides including aliphatic, unsaturated, and aromatic. Aliphatic polyanhydrides degrade in a few days while some aromatic polyanhydrides degrade over few years. Degradation rates of copolymers of aliphatic and aromatic polyanhydrides vary between these extremes and this feature of polyanhydrides gives an opportunity for making a drug delivery system which can provide the release of drugs for a desired time length of treatment [24].

The most frequently used is a copolymer of the 1,3bis(carboxyphenoxypropane) (PCPP) and sebacic acid (SA). PCPP is aromatic and hydrophobic and by itself has a long lifetime of over 3 years, while SA is aliphatic and hydrophilic with a lifetime of a few days. Copolymerization with SA reduces the lifetime to a few days [25]. The $80: 20$ copolymer has been FDA approved for intracranial delivery of carmustine (Gliadel) for treatment of brain tumors, and intravenous delivery for treatment of recurrent Hodgkin's lymphoma and multiple myeloma. In ocular use they have been investigated in the delivery of 5-fluorouracil, taxol, and etoposide for experimental glaucoma filtration surgery in a non-human primate model [26, 27]. 


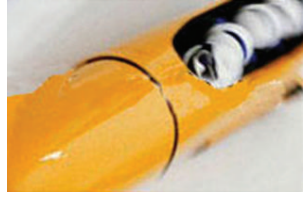

(a)

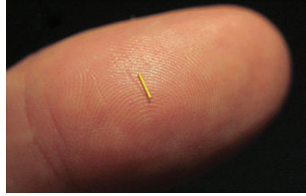

(c)

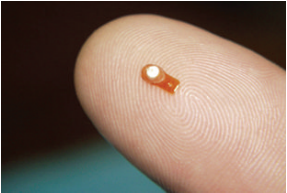

(b)

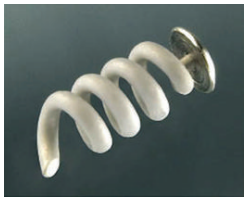

(d)

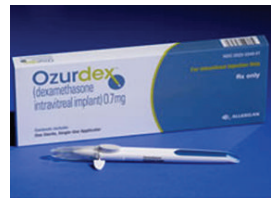

(e)

FIgure 1: Intravitreal devices. (a) Vitrasert, image courtesy of Bausch \& Lomb. (b) Retisert, image courtesy of pSIVIDA. (c) Medidur, image courtesy of pSIVIDA. (d) I-vation, image courtesy of SurModics, Inc. (e) Ozurdex, image courtesy of Allergan, Inc.

\subsection{Clinically Used Intravitreal Implants}

2.3.1. Ganciclovir. Vitrasert (Bausch \& Lomb, Rochester, NY) is a PVA-EVA reservoir implant consisting of a pellet containing at least $4.5 \mathrm{mg}$ of ganciclovir as the active ingredient and $0.25 \%$ magnesium stearate as the inactive ingredient with a ganciclovir release rate of $1 \mathrm{mcg} /$ hour (Figure 1(a)). The EVA limits the surface area of ganciclovir. A 5-6 mm scleral incision is made at pars plana and after trimming away any prolapsed vitreous, the device is implanted into the vitreous cavity. It is sutured in place on the sclera prior to closing the sclera and overlying conjunctiva. It is removed if another ganciclovir implant is placed (usually after 6 months) or if there are any complications such as endophthalmitis or retinal detachment. Vitrasert offers superior control of retinitis over systemic ganciclovir therapy [28]. The Vitrasert disc is composed of outer and inner permeable PVA layers surrounding a discontinuous hydrophobic EVA film. The device allows diffusion of fluid into the device dissolving the drug pellet, which then diffuses into the vitreous at a constant rate [2].

2.3.2. Fluocinolone Acetonide. Retisert (Bausch \& Lomb, Rochester, NY) is a tablet containing $0.59 \mathrm{mg}$ of fluocinolone acetonide that is coated with nonbiodegradable PVA and silicon laminate (Figure 1(b)). It is $5 \mathrm{~mm}$ long, $2 \mathrm{~mm}$ wide and $1.5 \mathrm{~mm}$ thick with a release rate of $0.3-0.6 \mathrm{mcg} / \mathrm{d}$ over a period of 30 months. It is inserted into the vitreous cavity and sutured to the sclera through a pars plana surgical technique similar to Vitrasert. In April 2005 it became the first FDA-approved device for use in the treatment of chronic noninfectious posterior uveitis [29].
In clinical studies Retisert was found to significantly reduce inflammation and lower intravitreal vascular endothelial growth factor (VEGF) levels. In patients with noninfectious posterior uveitis treated with Retisert, the recurrence rate of uveitis was reduced from $62 \%$ before treatment to $4 \%, 10 \%$, and $20 \%$ at 1,2 , and 3 years, respectively following treatment $[30,31]$. Despite the excellent reduction of uveitis, the complication rate was high. At 34 weeks, $51 \%$ of patients had an increased intraocular pressure (IOP) that required pressure lowering agents. At 3 years, $78 \%$ required pressure lowering agents and approximately $40 \%$ required glaucoma filtering surgery. In addition, 100\% of phakic patients developed cataract formation within 3 years of implantation. Other side effects included hypotony (6.1\%), retinal detachment (2.9\%), endophthalmitis $(0.4 \%)$, and the need for explantation at 2 years $(3.6 \%)[31,32]$.

Recently, the Multicenter Uveitis Steroid Treatment (MUST) trial compared the relative effectiveness of systemic therapy and fluocinolone acetonide implant for the treatment of noninfectious uveitis in 479 eyes over 2 years. It found that both treatment groups were effective and neither group was superior to the other in improving visual acuity. Systemic therapy was well tolerated while the implant group had an $80 \%$ risk of cataract surgery and $61 \%$ required treatment for elevated intraocular pressures [33].

2.3.3. Iluvien (Fluocinolone Acetonide). Illuvien (Alimera Sciences Inc., Alpharetta, GA; pSivida Inc., Watertown, $\mathrm{MA}$ ) is an injectable nonbiodegradable intravitreal implant containing fluocinolone acetonide (Figure $1(\mathrm{c})$ ). It is $3.5 \mathrm{~mm}$ long and $0.37 \mathrm{~mm}$ wide and releases fluocinolone acetonide at a rate of $0.2 \mathrm{mcg}$ or $0.5 \mathrm{mcg}$ per day over $18-36$ months. It is inserted with a 25 gauge needle. Phase III clinical trials for diabetic macular edema (DME) were recently concluded.

The fluocinolone acetonide for diabetic macular edema (FAME) study group tested the low dose $0.2 \mathrm{mcg}$ insert and the high dose $0.5 \mathrm{mcg}$ insert against a sham implant. At 24 months, $28 \%$ of those receiving either dose had an improvement of $\geq 15$ in best-corrected visual acuity (BCVA) letters compared to $16 \%$ of those in the control. At 36 months, it was 33.0\% in the low dose and $31.9 \%$ in the high dose compared with $21.4 \%$ in the sham. Increased incidence of cataracts was seen in implanted eyes but long-term vision was not compromised. Increase in intraocular pressure was also a concern with implantation of the device. At 36 months, $4.8 \%$ of those receiving the low dose implant required glaucoma surgery but visual outcome was not impacted when compared to those who did not require incisional surgery. These results show promise in DME patients who otherwise have limited effective treatment options [34]. In addition to its use in DME patients, phase II trials for the treatment of exudative age-related macular degeneration (AMD) and retinal vein occlusion are also being conducted.

\subsubsection{Triamcinolone Acetonide}

(1) I-vation. (SurModics, Eden Prairie, MN) is a $0.4 \mathrm{~mm} \times$ $0.21 \mathrm{~mm}$ titanium helical nonbiodegradable implant that 
TABLE 3: Comparison of corticosteroid properties.

\begin{tabular}{lccc}
\hline Steroid & $\begin{array}{c}\text { Water solubility } \\
(\mu / \mathrm{mL})\end{array}$ & Half-life & $\begin{array}{c}\text { Relative } \\
\text { potency }\end{array}$ \\
\hline Triamcinolone acetonide & 21 & 18 days & 1 \\
Fluocinolone acetonide & 50 & $1.3-1.7$ hours & $0.4 x$ \\
Dexamethasone & 100 & $3-5$ hours & $3-5 x$ \\
\hline
\end{tabular}

contains $0.925 \mathrm{mcg}$ triamcinolone acetonide (Figure $1(\mathrm{~d})$ ). Triamcinolone is coated with polybutyl methacrylate and polyEVA. It is intended for a sustained delivery of 2 years. The helical design increases the surface area for drug release and stabilizes the device onto the sclera [35]. It was recently found to be effective in the treatment of diabetic macular edema after 24 months in a Phase I clinical trial although all phakic patients developed visually significant cataracts and increases in intraocular pressure occurred in $50 \%$ of eyes [36].

(2) Verisome. Verisome (Ramscor, Inc., Menlo Park, CA) is a nonpolymer-based intraocular drug delivery system that provides long-acting intravitreal drug therapy. It can be injected through the pars plana using a 30 gauge injector. Triamcinolone has been used investigationally providing a mean vitreous level of $1.1 \mathrm{mcg} / \mathrm{mL}$ for up to 1 year [37].

\subsubsection{Dexamethasone}

(1) Ozurdex. Ozurdex (formerly Posurdex Allergan Inc., Irvine CA) is a rod-shaped $6.5 \times 0.45 \mathrm{~mm}$ pellet composed of a mixture of dexamethasone as the active pharmaceutical ingredient (API) and biodegradable PLGA (Figure 1(e)). Although dexamethasone has a short half-life relative to triamcinolone, it is 20 and 5 times more potent than fluocinolone and triamcinolone, respectively (Table 3) [38].

Ozurdex is placed intravitreally through the pars plana with an injector using a 22-gauge needle device. The insert contains $0.7 \mathrm{mg}$ dexamethasone and provides peak doses for 2 months initially followed by lower doses for up to 6 months. Ozurdex received FDA approval in June 2009 for the treatment of macular edema associated with retinal vein occlusion, and in September 2010, it became the second FDA-approved therapeutic agent for the treatment of noninfectious posterior uveitis.

In a 26-week, multicenter, double-masked, randomized clinical study in which 229 patients were randomized in a $1: 1: 1$ ratio receiving $0.70 \mathrm{mg}$ Ozurdex $(n=77), 0.35 \mathrm{mg}$ Ozurdex $(n=76)$, or sham injection $(n=76)$. Eighty-one percent of patients had intermediate uveitis. At the eighth week primary endpoint, $47 \%, 36 \%$ and $12 \%$ of patients had no vitreous inflammation. The response was maintained at week 26. In addition, both treatment groups achieved a 3-line improvement in visual acuity and reduced central macular thicknesses on ocular coherence tomography at 8 weeks that was statistically significant compared to the sham group. The complication rates were not found to be significant. Twentythree percent of eyes in the $0.7 \mathrm{mg}$ Ozurdex group required IOP-lowering agents and none needed surgical intervention for glaucoma. Cataract formation was seen in $15 \%$ in the $0.7 \mathrm{mg}$ group, $12 \%$ in the $0.34 \mathrm{mg}$ group, and $7 \%$ in the sham group $[39,40]$.

(2) Surodex. Surodex (Oculex Pharmaceuticals, Sunnyvale, $\mathrm{CA}$ ) is a $1.0 \times 0.4 \mathrm{~mm}$ PLGA pellet that provides sustained release of dexamethasone after insertion into the anterior chamber. It is primarily targeted to reduce post-cataract surgery inflammation for 7-10 days [41].

\subsection{Experimental Intravitreal Implants}

2.4.1. Cyclosporine. Cyclosporine A placed in the deep sclera adjacent to the suprachoroidal space has been found to be effective in controlling uveitis in an equine recurrent uveitis model [42]. In a chronic uveitis rabbit model, $2 \mathrm{mg}$ cyclosporine A conjugated to a PCL/PLGA copolymer was found to be significantly more effective than oral cyclosporine [20].

2.4.2. Indomethacin. PLGA discs containing $7 \mathrm{mg}$ of indomethacin released over 3 weeks was evaluated in a rabbit model. Although postoperative inflammation was decreased there was no significant decrease in posterior capsular opacification of the lens [43].

2.4.3. Particulate Drug Delivery Systems. The long-term drug delivery of small scale biodegradable devices has been recently investigated in experimental studies. These include liposomes, microspheres, and nanoparticles.

(1) Liposomes. Liposomes are spherical liposomal structures, about 0.01 to $10 \mu \mathrm{m}$ in diameter (Figure 2). They are formed of a vesicular lipid bilayer separated by water or an aqueous buffer compartment [44]. They can circumvent cell membrane barriers and protect drugs from metabolic or immune attack. Since the phospholipid bilayers are naturally occurring, they are biocompatible and minimize toxicity and immunogenicity.

Liposomes are colloidal particles made of phospholipids that encapsulate hydrophobic or hydrophilic therapeutic agents. They often contain inner aqueous spaces where hydrophilic enzymes remain soluble and hydrophobic outer layers that allow passage through natural membrane barriers.

Currently verteporfin (Visudyne, QLT Inc. Vancouver $\mathrm{BC}$, Canada) is the only liposomal drug that is FDA approved for use in the eye for the treatment of predominantly classic wet AMD. Liposomal amphoterecin B (AmBisome, Gilead Sciences, Foster City, CA) is used off-label for the treatment of fungal endophthalmitis and has been found to exhibit fewer side effects than the nonliposomal forms allowing for higher dosages (up to 30 micrograms) to be injected intravitreally [45].

(2) Particulate Ocular Drug Delivery Systems. Particulate Ocular Drug Delivery Systems include nanoparticles and microparticles. Although the distinction is often not consistent, nanoparticles are considered to be between 10 and 
TABLE 4: Comparison of intravitreal implants for the treatment of noninfectious uveitis [40].

\begin{tabular}{lcc}
\hline & $0.59 \mathrm{mg}$ fluocinolone acetonide (FA) implant & 0.7 mg dexamethasone (DEX) implant \\
\hline Administration & Operating room & Officebased \\
Matrix & Non-biodegradable & Biodegradable \\
Duration of effect & 30 months & 6 months \\
Improvement of $>15$ letters ( $\%$ eyes) & $21 \%$ by week 34 & $38 \%$ by week 26 \\
Rescue medications ( $\%$ eyes) & $25.4 \%$ by week 34 & $22 \%$ by week 26 \\
Glaucoma surgery ( $\%$ eyes) & $30.6 \%$ by month 24 & $0.5 \%$ by month 6 \\
Cataract surgery ( $\%$ eyes) & $89.4 \%$ by month 24 & $4 \%$ by month 6 \\
\hline
\end{tabular}

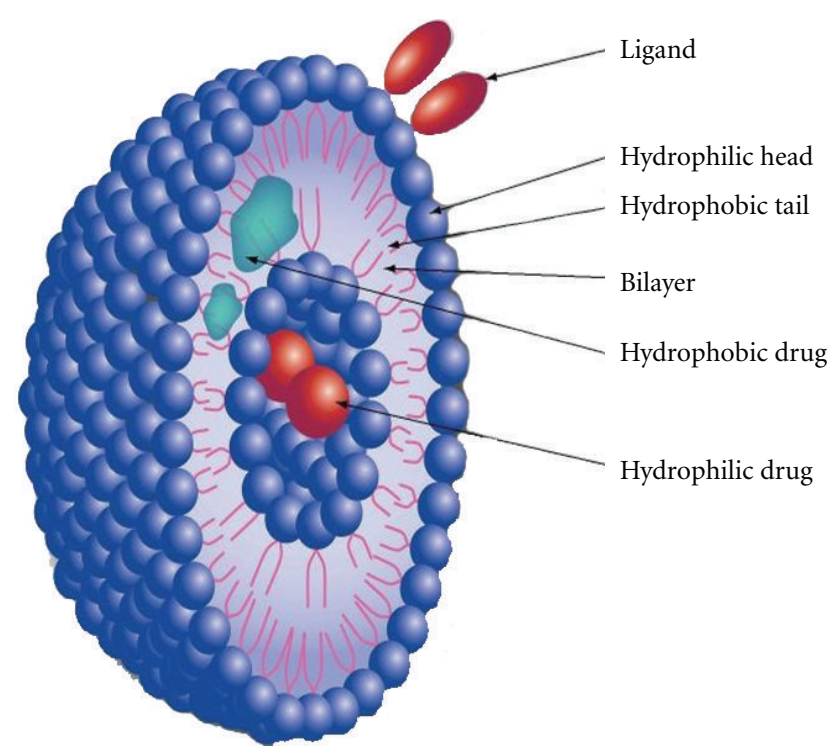

Figure 2: Liposome and its different drug-loading and surface functionalization modalities. (Courtesy of Nanomedicine (2010) Future Medicine Ltd).

$1,000 \mathrm{~nm}$ in size and microparticles 1 to $1,000 \mu \mathrm{m}$ in diameter [46]. Nanoparticles and microparticles are subdivided into nanospheres and microspheres which are a polymer-drug combinations where the drug is homogenously dispersed in the polymeric matrix, and nanocapsules/microcapsules, in which the drug particles or droplets are entrapped in a polymeric membrane (Figure 3 ).

(3) Microparticles. Microparticles are similar to liposomes in shape, size, and route of administration. However, nanoparticles offer several advantages over microsomes such as higher stability and larger drug-loading capabilities. Polymers such as PLGA and PLA are widely used for nanoparticle drug delivery systems. Surface polymer modifications also provide greater protection of the drug against degradation and phagocytosis by macrophages [47]. Although there are no currently used FDA approved microparticle devices, a wide variety of therapeutic agents are being investigated to improve the cellular penetration and allow long-term delivery using microsphere and nanosphere technology (Table 4).

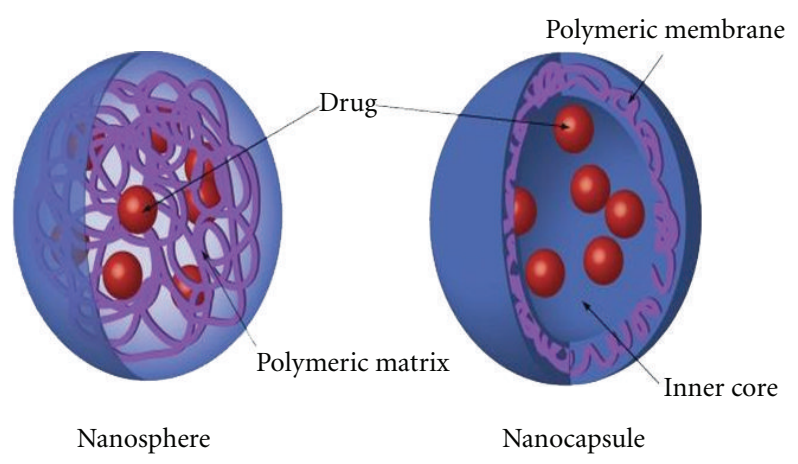

FIGURE 3: The two main types of polymeric nanoparticles known as nanosphere (matrix system) and nanocapsule (reservoir system) with different drug-loading modalities. (Courtesy of Nanomedicine (2010) Future Medicine Ltd).

(4) Microspheres. Microspheres have been developed for sustained ocular delivery of therapeutic agents such as progesterone, adriamycin, and pegaptanib [48-50]. Microspheres composed of chitosan, a natural biodegradable polymer, have been used for transcorneal acyclovir delivery [51]. A sustained release of microsphere-encapsulated cyclosporine was found to be present compared to cyclosporine solution [52].

(5) Nanoparticles. Nanoparticles have been used experimentally with several agents. Tamoxifen (PEG coated) was found to be effective in the treatment of experimental autoimmune uveitis in a rat model [53]. Intravitreally injected nanoparticles containing ganciclovir and acyclovir have been studied in a rabbit model with steady drug concentrations, but were found to be associated with cataract formation and flare [54]. Scleral injections of pigment epitheliumderived factor (PEDF) resulted in increased PEDF expression in the retina and retinal pigment epithelium and resulted in significant reductions of choroidal neovascularization in mouse and pig models $[55,56]$. In a Phase I clinical trial, recombinant adeno-associated viral mediated expression of (rAAV-PEDF) was administered intravitreally in patients with exudative AMD. Although this resulted in transient intraocular inflammation (25\%) and IOP elevations (21\%), no other adverse events were seen and the majority of the patients achieved stable or improved visual acuity [57]. 


\section{Summary}

In conclusion, the eye is well suited for local delivery of therapeutics to treat vitreous inflammation as well as other pathologic conditions that induce visual loss. However, there are some unique challenges in designing local ocular drug delivery devices, which include implants, polymers, and micro- and nanoparticles. An integrated approach involving biomedical engineering, molecular biology, immunology, pathology, and pharmacology will continue to be critical to designing optimal devices for ocular inflammation and other diseases.

\section{References}

[1] S. Duvvuri, S. Majumdar, and A. K. Mitra, "Drug delivery to the retina: challenges and opportunities," Expert Opinion on Biological Therapy, vol. 3, no. 1, pp. 45-56, 2003.

[2] T. J. Smith, P. A. Pearson, D. L. Blandford et al., "Intravitreal sustained-release ganciclovir," Archives of Ophthalmology, vol. 110, no. 2, pp. 255-258, 1992.

[3] S. S. Lee, P. Hughes, A. D. Ross, and M. R. Robinson, "Biodegradable implants for sustained drug release in the eye," Pharmaceutical Research, vol. 27, no. 10, pp. 2043-2053, 2010.

[4] J. I. Lim, R. A. Wolitz, A. H. Dowling, H. R. Bloom, A. R. Irvine, and D. M. Schwartz, "Visual and anatomic outcomes associated with posterior segment complications after ganciclovir implant procedures in patients with AIDS and cytomegalovirus retinitis," American Journal of Ophthalmology, vol. 127, no. 3, pp. 288-293, 1999.

[5] D. L. Blandford, T. J. Smith, J. D. Brown, P. A. Pearson, and P. Ashton, "Subconjunctival sustained release 5-fluorouracil," Investigative Ophthalmology and Visual Science, vol. 33, no. 12, pp. 3430-3435, 1992.

[6] C. K. Cheng, A. S. Berger, P. A. Pearson, P. Ashton, and G. J. Jaffe, "Intravitreal sustained-release dexamethasone device in the treatment of experimental uveitis," Investigative Ophthalmology and Visual Science, vol. 36, no. 2, pp. 442-453, 1995.

[7] P. A. Pearson, G. J. Jaffe, D. F. Martin et al., "Evaluation of a delivery system providing long-term release of cyclosporine," Archives of Ophthalmology, vol. 114, no. 3, pp. 311-317, 1996.

[8] M. H. Rahimy, G. A. Peyman, S. Y. Chin et al., "Polysulfone capillary fiber for intraocular drug delivery: in vitro and in vivo evaluations," Journal of Drug Targeting, vol. 2, no. 4, pp. 289-298, 1994.

[9] M. H. Rahimy, G. A. Peyman, M. L. Fernandes, S. H. El-Sayed, Q. Luo, and H. Borhani, "Effects of an intravitreal daunomycin implant on experimental proliferative vitreoretinopathy: simultaneous pharmacokinetic and pharmacodynamic evaluations," Journal of Ocular Pharmacology, vol. 10, no. 3, pp. 561-570, 1994.

[10] R. Jain, N. H. Shah, A. W. Malick, and C. T. Rhodes, "Controlled drug delivery by biodegradable poly(ester) devices: different preparative approaches," Drug Development and Industrial Pharmacy, vol. 24, no. 8, pp. 703-727, 1998.

[11] E. Bowland, G. Wnek, and G. Bowlin, "Poly(glycolic acid)," in Encyclopedia of Biomaterials and Biomedical Engineering, G. E. Wnek and G. L. Bowlin, Eds., pp. 2241-2248, Marcel Dekker, New York, NY, USA, 2008.

[12] M. S. Shive and J. M. Anderson, "Biodegradation and biocompatibility of PLA and PLGA microspheres," Advanced Drug Delivery Reviews, vol. 28, no. 1, pp. 5-24, 1997.
[13] T. Yasukawa, H. Kimura, N. Kunou et al., "Biodegradable scleral implant for intravitreal controlled release of ganciclovir," Graefe's Archive for Clinical and Experimental Ophthalmology, vol. 238, no. 2, pp. 186-190, 2000.

[14] N. Kunou, Y. Ogura, T. Yasukawa et al., "Long-term sustained release of ganciclovir from biodegradable scleral implant for the treatment of cytomegalovirus retinitis," Journal of Controlled Release, vol. 68, no. 2, pp. 263-271, 2000.

[15] C. Pitt, "Poly-e-caprolactone and its copolymers," in Biodegradable Polymers as Drug Delivery Systems, M. Chasin and R. S. Langer, Eds., p. 71, Marcel Dekker, New York, NY, USA, 1990.

[16] G. A. Peyman, B. Khoobehi, S. Shaibani, S. Shamsnia, and I. Ribeiro, "A fluorescent vesicle system for the measurement of blood velocity in the choroidal vessels," Ophthalmic Surgery and Lasers, vol. 27, no. 6, pp. 459-466, 1996.

[17] J. S. Chawla and M. M. Amiji, "Biodegradable poly $(\varepsilon-$ caprolactone) nanoparticles for tumor-targeted delivery of tamoxifen," International Journal of Pharmaceutics, vol. 249, no. 1-2, pp. 127-138, 2002.

[18] H. Borhani, G. A. Peyman, M. H. Rahimy, and H. Thompson, "Suppression of experimental proliferative vitreoretinopathy by sustained intraocular delivery of 5-FU," International Ophthalmology, vol. 19, no. 1, pp. 43-49, 1995.

[19] A. Silva-Cunha, S. L. Fialho, M. C. Naud, and F. Behar-Cohen, "Poly-epsilon-caprolactone intravitreous devices: an in vivo study," Investigative Ophthalmology and Visual Science, vol. 50, no. 5, pp. 2312-2318, 2009.

[20] N. R. F. Beeley, J. V. Rossi, P. A. A. Mello-Filho et al., "Fabrication, implantation, elution, and retrieval of a steroid-loaded polycaprolactone subretinal implant," Journal of Biomedical Materials Research Part A, vol. 73, no. 4, pp. 437-444, 2005.

[21] X. Dong, W. Shi, G. Yuan, L. Xie, S. Wang, and P. Lin, "Intravitreal implantation of the biodegradable cyclosporin A drug delivery system for experimental chronic uveitis," Graefe's Archive for Clinical and Experimental Ophthalmology, vol. 244, no. 4, pp. 492-497, 2006.

[22] W. Shi, T. Liu, L. Xie, and S. Wang, "FK506 in a biodegradable glycolide-co-clatide-co-caprolactone polymer for prolongation of corneal allograft survival," Current Eye Research, vol. 30, no. 11, pp. 969-976, 2005.

[23] K. E. Uhrich, S. M. Cannizzaro, R. S. Langer, and K. M. Shakesheff, "Polymeric systems for controlled drug release," Chemical Reviews, vol. 99, no. 11, pp. 3181-3198, 1999.

[24] J. P. Jain, S. Modi, A. J. Domb, and N. Kumar, "Role of polyanhydrides as localized drug carriers," Journal of Controlled Release, vol. 103, no. 3, pp. 541-563, 2005.

[25] K. W. Leong, V. Simonte, and R. Langer, "Synthesis of polyanhydrides: melt-polycondensation, dehydrochlorination, and dehydrative coupling," Macromolecules, vol. 20, no. 4, pp. 705$712,1987$.

[26] H. D. Jampel, K. W. Leong, G. R. Dunkelburger, and H. A. Quigley, "Glaucoma filtration surgery in monkeys using 5fluorouridine in polyanhydride disks," Archives of Ophthalmology, vol. 108, no. 3, pp. 430-435, 1990.

[27] H. D. Jampel, P. Koya, K. Leong, and H. A. Quigley, "In vitro release of hydrophobic drugs from polyanhydride disks," Ophthalmic Surgery, vol. 22, no. 11, pp. 676-680, 1991.

[28] D. F. Martin, D. J. Parks, S. D. Mellow et al., "Treatment of cytomegalovirus retinitis with an intraocular sustainedrelease ganciclovir implant: a randomized controlled clinical trial," Archives of Ophthalmology, vol. 112, no. 12, pp. 15311539, 1994. 
[29] G. J. Jaffe, J. Ben-nun, H. Guo, J. P. Dunn, and P. Ashton, "Fluocinolone acetonide sustained drug delivery devine to treat severe uveitis," Ophthalmology, vol. 107, no. 11, pp. 20242033, 2000.

[30] G. J. Jaffe, D. Martin, D. Callanan, P. A. Pearson, B. Levy, and T. Comstock, "Fluocinolone acetonide implant (retisert) for noninfectious posterior uveitis. Thirty-four-week results of a multicenter randomized clinical study," Ophthalmology, vol. 113, no. 6, pp. 1020-1027, 2006.

[31] D. G. Callanan, G. J. Jaffe, D. F. Martin, P. A. Pearson, and T. L. Comstock, "Treatment of posterior uveitis with a fluocinolone acetonide implant: three-year clinical trial results," Archives of Ophthalmology, vol. 126, no. 9, pp. 1191-1201, 2008.

[32] D. A. Goldstein, D. G. Godfrey, A. Hall et al., "Intraocular pressure in patients with uveitis treated with fluocinolone acetonide implants," Archives of Ophthalmology, vol. 125, no. 11, pp. 1478-1485, 2007.

[33] J. H. Kempen, M. M. Altaweel, J. T. Holbrook et al., "Randomized comparison of systemic anti-inflammatory therapy versus fluocinolone acetonide implant for intermediate, posterior, and panuveitis: the multicenter uveitis steroid treatment trial," Ophthalmology, vol. 118, no. 10, pp. 1916-1926, 2011.

[34] P. A. Campochiaro, D. M. Brown, A. Pearson et al., "Sustained delivery fluocinolone acetonide vitreous inserts provide benefit for at least 3 years in patients with diabetic macular edema," Ophthalmology. In press.

[35] "SurModics I-vationTM Sustained Drug Delivery System: Intravitreal Implant for Drug Delivery," 2010, http://www .surmodics.com/pdf/ophthalmology.pdf.

[36] P. U. Dugel, D. Eliott, H. L. Cantrill et al., "I-Vation TA: 24month clinical results of the Phase I safety and preliminary efficacy study," in Proceedings of ARVO Annual Meeting, Fort Lauderdale, Fla, USA, 2009.

[37] M. Hu, G. Huang, F. Karasina, and V. G. Wong, "Verisome, a novel injectable, sustained release, biodegradable, intraocular drug delivery system and triamcinolone acetonide," Investigative Ophthalmology and Visual Science, vol. 49, E-Abstract 5627, 2008.

[38] J. L. Edelman, "Differentiating intraocular glucocorticoids," Ophthalmologica, vol. 224, no. 1, supplement, pp. 25-30, 2010.

[39] C. Lowder, R. Belfort, S. Lightman et al., "Dexamethasone intravitreal implant for noninfectious intermediate or posterior uveitis," Archives of Ophthalmology, vol. 129, no. 5, pp. 545-553, 2011.

[40] R. S. Hunter and A. M. Lobo, "Dexamethasone intravitreal implant for the treatment of noninfectious uveitis," Clinical Ophthalmology, vol. 5, pp. 1613-1621, 2011.

[41] D. T. H. Tan, S. P. Chee, L. Lim, and A. S. M. Lim, "Randomized clinical trial of a new dexamethasone delivery system (Surodex) for treatment of post-cataract surgery inflammation," Ophthalmology, vol. 106, no. 2, pp. 223-231, 1999.

[42] B. C. Gilger, J. H. Salmon, D. A. Wilkie et al., "A novel bioerodible deep scleral lamellar cyclosporine implant for uveitis," Investigative Ophthalmology and Visual Science, vol. 47, no. 6, pp. 2596-2605, 2006.

[43] O. Nishi, K. Nishi, T. Morita, Y. Tada, E. Shirasawa, and K. Sakanishi, "Effect of intraocular sustained release of indomethacin on postoperative inflammation and posterior capsule opacification," Journal of Cataract and Refractive Surgery, vol. 22, supplement, pp. 806-810, 1996.

[44] D. Ghate and H. F. Edelhauser, "Ocular drug delivery," Expert Opinion on Drug Delivery, vol. 3, no. 2, pp. 275-287, 2006.
[45] M. Barza, J. Baum, and C. Tremblay, "Ocular toxicity of intravitreally injected liposomal amphotericin B in rhesus monkeys," American Journal of Ophthalmology, vol. 100, no. 2, pp. 259-263, 1985.

[46] J. Kreuter, "Nanoparticles," in Encyclopedia of Pharmaceutical Technology, J. Swarbrick and J. C. Boylan, Eds., pp. 165-190, Marcel Dekker, New York, NY, USA, 1994.

[47] C. Roney, P. Kulkarni, V. Arora et al., "Targeted nanoparticles for drug delivery through the blood-brain barrier for Alzheimer's disease," Journal of Controlled Release, vol. 108, no. 2-3, pp. 193-214, 2005.

[48] L. R. Beck, D. R. Cowsar, and D. H. Lewis, "A new longacting injectable microcapsule system for the administration of progesterone," Fertility and Sterility, vol. 31, no. 5, pp. 545$551,1979$.

[49] T. Moritera, Y. Ogura, N. Yoshimura et al., "Biodegradable microspheres containing adriamycin in the treatment of proliferative vitreoretinopathy," Investigative Ophthalmology and Visual Science, vol. 33, no. 11, pp. 3125-3130, 1992.

[50] K. G. Carrasquillo, J. A. Ricker, I. K. Rigas, J. W. Miller, E. S. Gragoudas, and A. P. Adamis, "Controlled delivery of the anti-VEGF aptamer EYE001 with poly(lactic-co-glycolic)acid microspheres," Investigative Ophthalmology and Visual Science, vol. 44, no. 1, pp. 290-299, 2003.

[51] I. Genta, B. Conti, P. Perugini, F. Pavanetto, A. Spadaro, and G. Puglisi, "Bioadhesive microspheres for ophthalmic administration of acyclovir," Journal of Pharmacy and Pharmacology, vol. 49, no. 8, pp. 737-742, 1997.

[52] Y. He, Y. Liu, Y. Liu et al., "Cyclosporine-loaded microspheres for treatment of uveitis: in vitro characterization and in vivo pharmacokinetic study," Investigative Ophthalmology and Visual Science, vol. 47, no. 9, pp. 3983-3988, 2006.

[53] Y. de Kozak, K. Andrieux, H. Villarroya et al., "Intraocular injection of tamoxifen-loaded nanoparticles: a new treatment of experimental autoimmune uveoretinitis," European Journal of Immunology, vol. 34, no. 12, pp. 3702-3712, 2004.

[54] M. S. El-Samaligy, Y. Rojanasakul, J. F. Charlton, G. W. Weinstein, and J. K. Lim, "Ocular disposition of nanoencapsulated acyclovir and ganciclovir via intravitreal injection in rabbit's eye," Drug Delivery, vol. 3, no. 2, pp. 93-97, 1996.

[55] P. Gehlbach, A. M. Demetriades, S. Yamamoto et al., "Periocular injection of an adenoviral vector encoding pigment epithelium-derived factor inhibits choroidal neovascularization," Gene Therapy, vol. 10, no. 8, pp. 637-646, 2003.

[56] Y. Saishin, R. L. Silva, Y. Saishin et al., "Periocular gene transfer of pigment epithelium-derived factor inhibits choroidal neovascularization in a human-sized eye," Human Gene Therapy, vol. 16, no. 4, pp. 473-478, 2005.

[57] P. A. Campochiaro, Q. D. Nguyen, S. M. Shah et al., "Adenoviral vector-delivered pigment epithelium-derived factor for neovascular age-related macular degeneration: results of a phase I clinical trial," Human Gene Therapy, vol. 17, no. 2, pp. 167-176, 2006. 


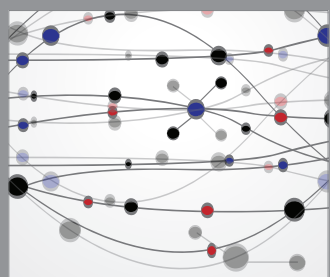

The Scientific World Journal
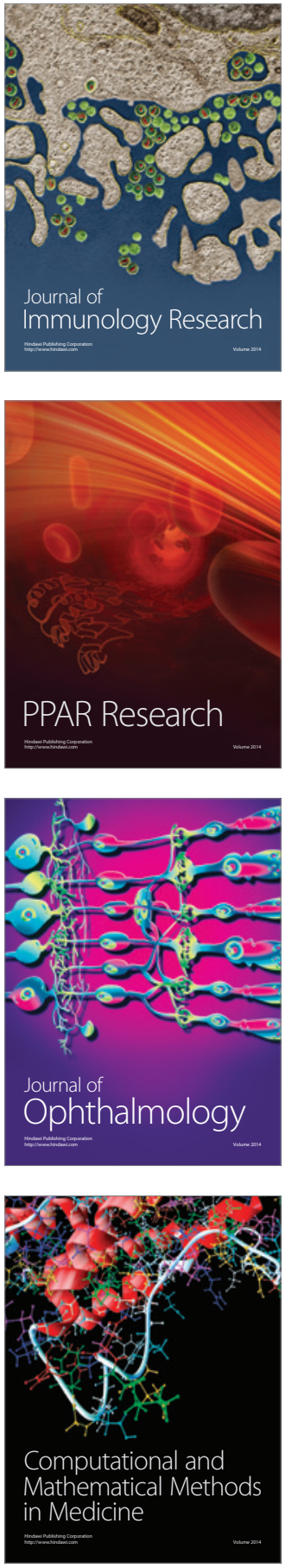

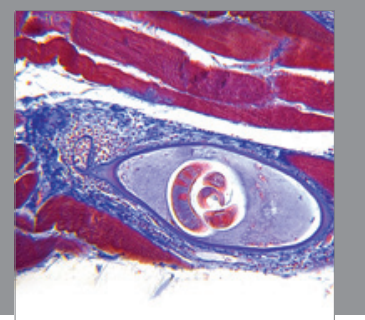

Gastroenterology

Research and Practice
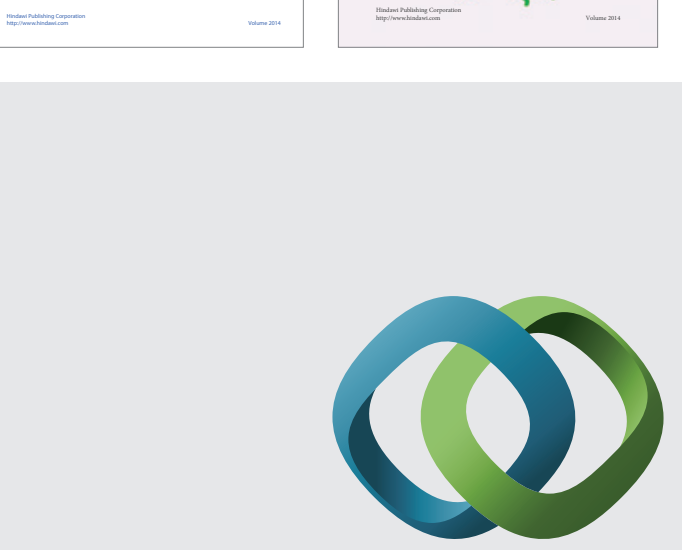

\section{Hindawi}

Submit your manuscripts at

http://www.hindawi.com
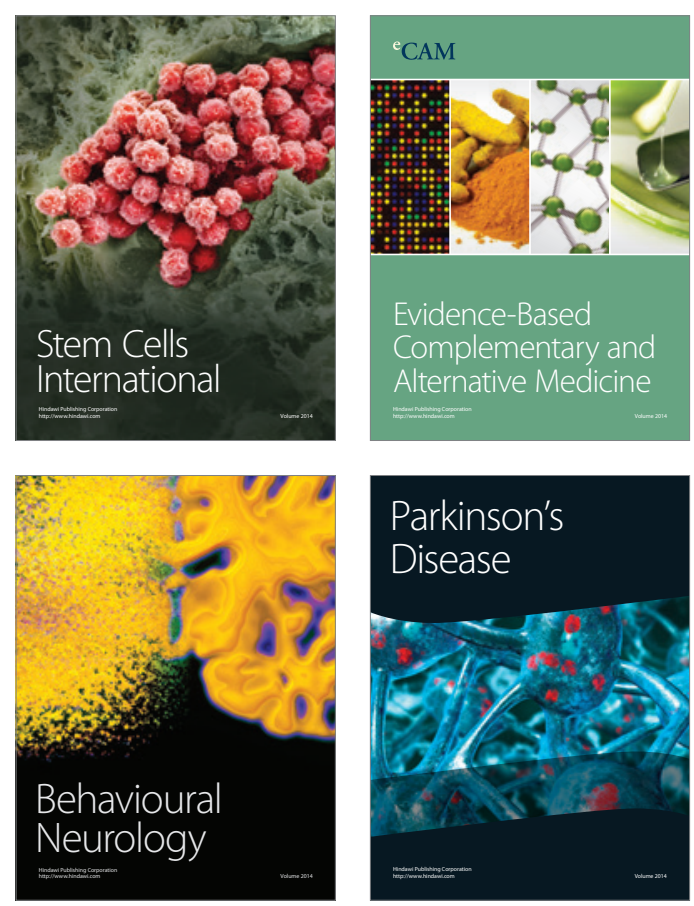

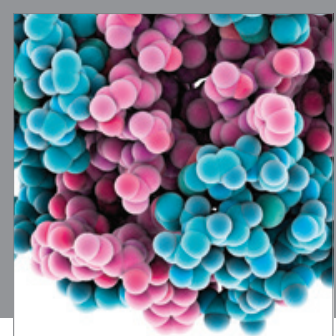

Journal of
Diabetes Research

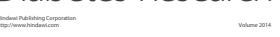

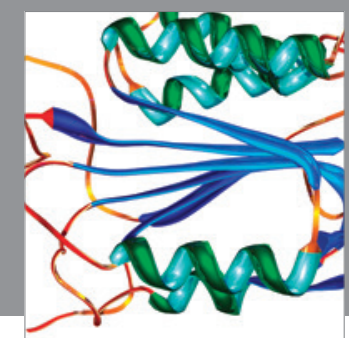

Disease Markers
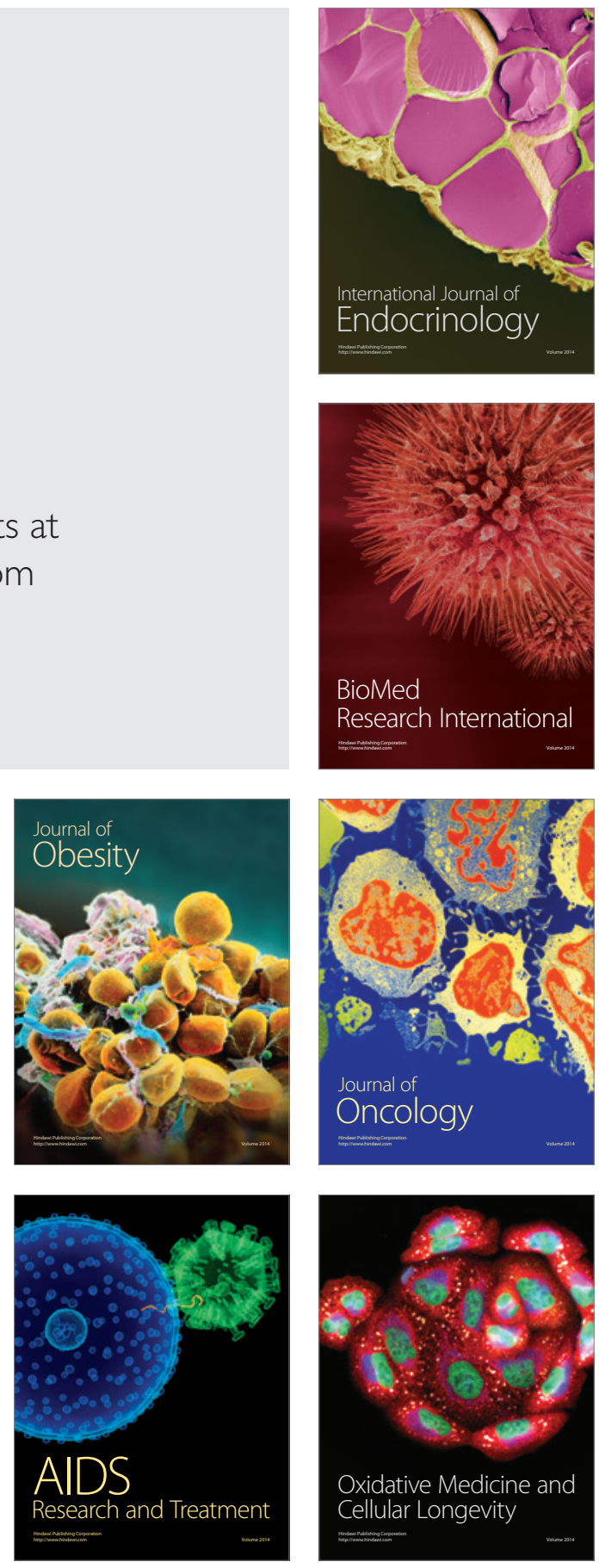